\title{
CREATING A PSEUDO SINGLE BUNCH AT THE ALS*
}

\author{
G. Portmann, S. Kwiatkowski, D. Plate, J. Julian, R. Low, K. Baptiste, W. Barry, D. Robin \\ Lawrence Berkeley National Laboratory (LBNL), Berkeley, CA 94720 U.S.A.
}

\begin{abstract}
Typically storage ring light sources operate with the maximum number of bunches as possible with a gap for ion clearing. By evenly distributing the beam current the overall beam lifetime is maximized. The Advanced Light Source (ALS) has 2 nanoseconds between the bunches and typically operates with 276 bunches out of a possible 328. For experimenters doing timing experiment this bunch separation is too small and would prefer to see only one or two bunches in the ring. The ALS allocates four weeks every year for dedicated 2-bunch operation. In order to provide more flexible operations and substantially increase the amount of operating time for time-of-flight experimenters, it is being proposed to kick one bunch on a different vertical closed orbit. By spatially separating the light from this bunch from the main bunch train in the beamline, one could potentially have single bunch operation all year round. By putting this bunch in the middle of the ion clearing gap the required bandwidth of the kicker magnets is reduced. Using one kicker magnet running at the ring repetition rate $(1.5 \mathrm{MHz})$, this bunch could be permanently put on a different closed orbit. Using multiple kicker magnets, this bunch could be locally offset at an arbitrary frequency.
\end{abstract}

\section{INTRODUCTION}

The concept of using a camshaft bunch started many years ago and originated out of the needs of time-of-flight experimenters. To our knowledge no accelerator in the world has taken the next step to kick the camshaft bunch on a different closed-orbit to create a pseudo single bunch mode. Accelerators like the APS and ESRF can achieve similar functionality by installing choppers in the beamlines. However, even with state-of-the-art choppers this solution requires relatively large gaps in the bunch train, so it's presently only feasible on large accelerators. It also requires each beamline to purchase a relatively expensive and often difficult to use and maintain chopper. At the ALS the largest gap in the bunch train is presently 104 nanoseconds, which is out of reach for x-ray choppers.

There are a number of beamlines at the ALS interested in exploring a pseudo single bunch operational mode. A major reason is so that experiments using the camshaft bunch will not have to use gated detectors. The ability to use integrating detectors increases the variety and quality of the experiments that can be done. For instances, the

\footnotetext{
* This work was supported U.S. Department of Energy under Contract No. DE-AC03-76SF00098 and DE-AC03-76SF00515.
}

02 Synchrotron Light Sources and FELs combination of the pseudo single bunch mode and a chopper with an open time of just more than one turn allows for an effective single bunch operation at 1-10 $\mathrm{kHz}$.

\section{POSSIBLE OPERATIONAL MODES}

By kicking the camshaft bunch on a different closedorbit, it may be possible to create a pseudo single bunch operation during a multi-bunch user run. There are a number of different ways the orbit of the camshaft bunch can be shaped depending on the number and location of the fast kicker magnets. The easiest thing to do is install one kicker magnet and place the camshaft bunch on a different global closed-orbit. This may not be optimal for all single bunch or multi-bunch users, but it would be a relatively easy thing to do to experiment with the method. Another obvious thing to do is locally bump the camshaft bunch in one part of the ring. This would isolate the disturbance to a relatively small section of the ring. A third option is to install kicker magnets all around the ring and profile the orbit much like global orbit correction.

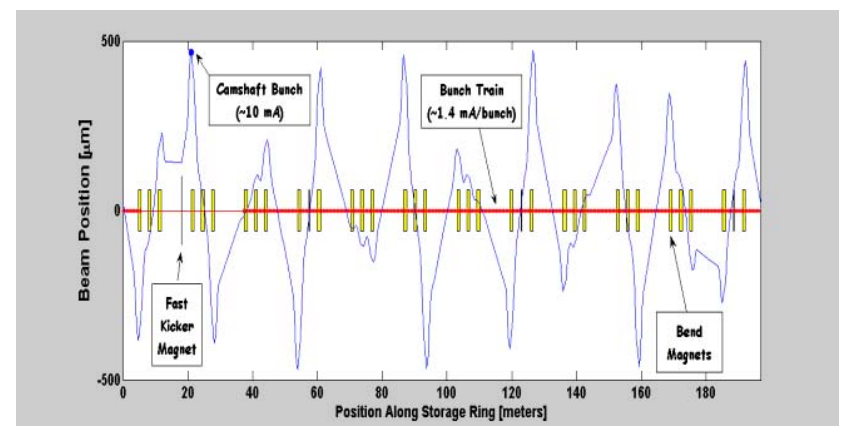

Fig. 1. Orbit Change for a One Kicker Magnet.

\section{GLOBAL ORBIT DISTORTIONS}

\section{Single Kicker Magnet}

The ALS is a 12 sector, triple bend achromat with 4.5 meter straight sections for insertion devices. Using 1 fast kicker magnet running at $1.5 \mathrm{MHz}$ the camshaft bunch can be permanently put on a different closed orbit. A convenient location to install a kicker happens to be in the straight section 2. Fig. 1 shows the change in the closed orbit for the camshaft bunch for a $60 \mu$ radian kick. As shown in the figure, this configuration would suitable for a number of beamlines. Many of the outer bend beamlines and some of the insertion device and center bends beamlines would see a sizeable separation. Fig. 2 is the same data with the beam sizes included. 


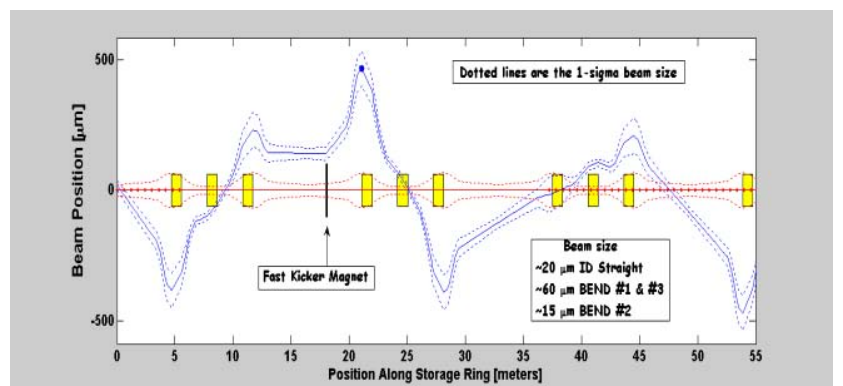

Fig. 2. 1- Kicker Magnet - First 3 Sectors.

\section{Reduce the Repetition Rate by $1.5 / \mathrm{n} \mathrm{MHz}$}

If a beamline is not a favorable location with respect to a single kicker magnet in Sector 2 (Fig. 1), it may not be the end of story. Being able to test this method on the bend magnet beamlines is extremely important. Instead of adding more kicker magnets, one can get interesting orbits by kicking at less than the revolution rate $-1.5 / 2$, $1.5 / 3,1.5 / 4$, etc. Fig. 3 shows the closed orbits for when kicking at $187.5 \mathrm{kHz}(\mathrm{n}=5)$. Notice that it takes 5-turns for the orbit to close.

The picture gets quite confusing if one skips too many turns before kicking, however, this method can extend the reach of a single kicker magnet. For instance, a user in straight section six sees mostly an angle change in Fig. 1 but will see reasonable positional changes if kicking every other turn or every fifth turn.

Since the vertical fractional tune in the ALS is .2, kicking the bump every fifth rotation will in resonance with the beam. Basically, the $1 / \sin (\pi v)$ term for a closed orbit change due to corrector blows up. If the tune was exactly .2, the bunch would be kicked out of the accelerator. However, if the tune is changed a small amount off .2, the kick will be just off resonance and a potential large amplification of the bump can be achieved. Fig. 4 shows the result for a vertical tune of 9.188 . This factor of 3 amplification comes from $1 / \sin (9.2 \pi)=-1.7$ and $1 / \sin (5 * 9.188 \pi)=-5.33$ or 3.14 times the kicker for free. There are two problems with using this method. First, all the different orbits in Fig. 3 may be a bit difficult to work with. That said, many of the beamlines would see one track which shows a large positional separation from all the other tracks. Second, the size of the orbit kick is directly dependent on the tune and the more amplification one tries to achieve the more sensitive it becomes to tune variation. For test purposes, one could also move the vertical tune to be on near resonance when kicking every 4 th turn.

Unfortunately kicking at too low a frequency will produce some interesting but probably not useful beam characteristics. When kicking relatively slowly, a transient will be introduced which defuses in phase space (beam blowup) then finally damps back to the closedorbit of the main bunch train.

The advantages of using a single magnet system is it takes less than $.5 \mathrm{~m}$ in the storage ring, it reaches a large number of users per kicker magnet, and it's relatively simple to operate. The disadvantage is it's a fixed frequency (1.5 MHz or a close fraction there of) and the fixed beam path may not be optimal for all users (or nonusers). The separation of the camshaft bunch from the main bunch train will vary depending on the beamline location.

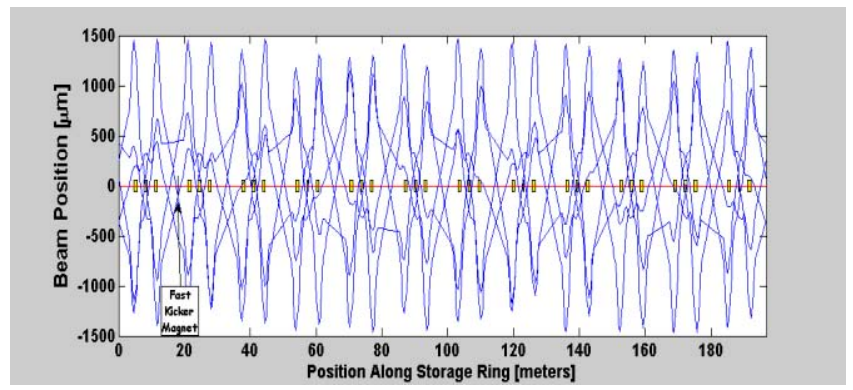

Fig. 3. Kick Every Fifth Turn.

\section{Global Orbit Profiling}

By adding more kicker magnets, one could more precisely shape the global orbit of the camshaft bunch. This is similar to global orbit correction except the corrector magnets only select one bunch.

If it's important to limit the separation of the camshaft from the main bunch train to a section of the accelerator, local bumps can be used. Three or four magnet bumps are easy to create and are commonly used in orbit control. The bump size could extend over a relatively small section, like an arc (Fig. 4) or a straight section, or it could extend over many sectors. For beamlines collecting data timed to a laser running at kilohertz frequencies, it is quite attractive to run the local bump at the same frequency.

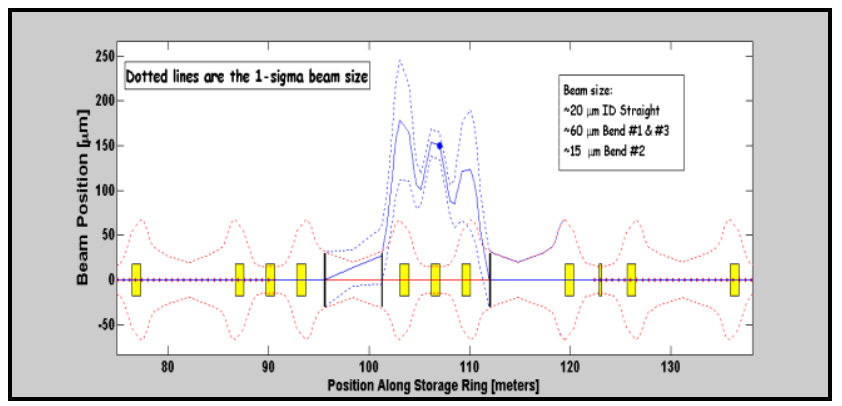

Fig. 4. Arc Sector Bump.

\section{BEAMLINE USER INPUT}

Before the final specification can be put together, more input is needed from the user community. The three basic issues are kick size (displacement), repetition rate, and contamination. There are also potential negative effects on storage ring operations.

\section{Kick Size (displacement and/or angle)}

The kick size is the required displacement or angle separation of the kicked bunch from the main bunch train 
The present design calls for a 5-10 beam sigma displacement. This requirement could possibly be verified by scanning a closed-orbit bump in normal multibunch mode.

One of the challenges here is every beamline is different and the quality of the optics plays a big role in determining the required separation. Experimental data from BL 5.3.1 is shown in Fig. 5 for a beam sigma of .1 $\mathrm{mm}$. Due to imperfect optics the beam profile distribution is only Gaussian to about 3.5 sigma. So there is only nominal improvement in bunch purity by kicking the beam past about 5 sigma. This beamline is roughly looking for a $10^{-3}$ reduction of the signal from the main bunch train.

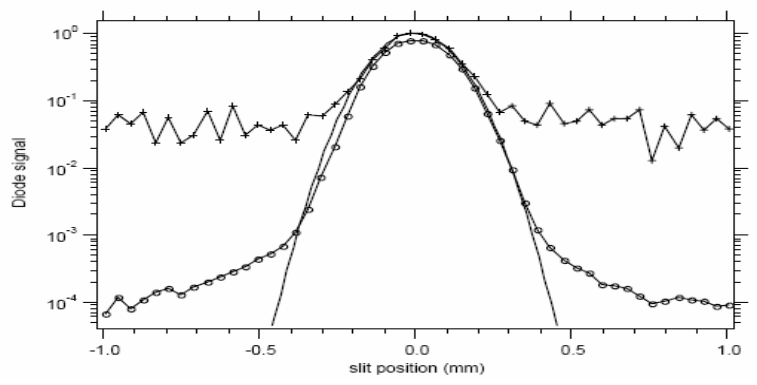

Fig. 5. Measured horizontal profile at BL 5.3.1 at 5 $\mathrm{keV}$. The crosses and circles represent two amplifier gains and the line without symbols shows a Gaussian fit (Data provided by Robert Schoenlein).

\section{Repetition Rate}

The upper bound on the repetition rate is revolution frequency of the electrons $-1.5 \mathrm{MHz}$. Certain user experiments may require slowly rates. For instance, experiments using lasers tend to be in the $1-10 \mathrm{kHz}$ range. When using local bumps it is possible to run at any fraction of the revolution rate.

\section{Contamination}

This is a serious question about whether or not the multi-bunch users can handle this new beam profile.

- Contamination effects due to changes in the background radiation (not a Gaussian beam profile anymore).

- Effects of small periodic changes to the beam current in the main bunch train in the local bump case with frequencies less than $1.5 \mathrm{MHz}$.

Understanding this area better needs attention. Potential storage ring operational problems also need to be studied. The orbit will change with the kick size and current of the camshaft bunch and non-repeatability of the kicker magnet would add noise to the bunch train and possibly a beam size increase due to diffusion.

\section{FAST KICKER MAGNET DESIGN}

The main parts of the fast kicker are the pulser and the magnet. The magnet will be a stripline kicker similar to the fast feedback kickers which were designed at the
ALS. The pulser circuit provides the voltage to the kicker. The goal of the pulser is to supply high voltage when the camshaft bunch is present and zero volts the rest of the time. There was some hope of purchasing the pulser from industry but the $1.5 \mathrm{MHz}$ requirement puts too big a heat load on all commercial units that were considered. For beamline requiring only $10 \mathrm{KHz}$ or less repartition rate, there may be an off-the-shelf commercial unit with fast enough rise/fall time to meet the specification. However, running less then $1.5 \mathrm{MHz}$ can only be done using local bumps.

A candidate design for the pulser running at $1.5 \mathrm{MHz}$ (or less) and providing a $60 \mu$ radian kick (1.15 KVolts). Fig. 6 shows the pulse shape. If the gap in the bunch train is reduced in the future, the length of the flat top will need to be reduced. It is unclear at this point if the $52 \mu$ radian kick can still be obtained with a smaller gap.

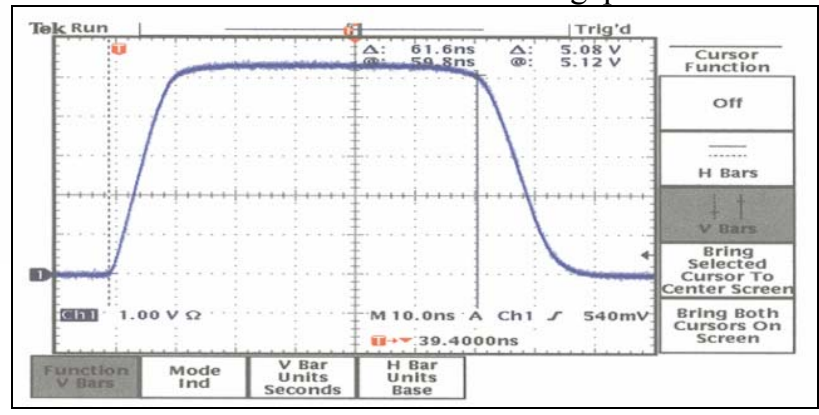

Fig. 6. Fast Kicker Pulse.

\section{A REAL TEST}

Depending on the operational mode and the location of the beamline with respect to the kick, there can be negative effects on beamlines using the multi-bunch beam. Since there are many beamlines with unique optics, it's difficult to predict the impact on every beamline. Since it will be nearly impossible to understand the benefits and possible problems for users and the accelerator without actually trying it, a prototype magnet is presently being design and should be ready for installation in 2007. This magnet will provide at least a $60 \mu$ radian kick to the electron beam at $1.9 \mathrm{GeV}$ at a repartition rate of $1.5 \mathrm{MHz}$ (or less). The main goal is to study the benefits and contamination issues of this method.

\section{ACKNOWLEDGEMENTS}

We would like to acknowledge Brian Kincaid who suggested this approach many years ago and Janos Kirz for providing the support and encouragement to pursue it.

\section{REFERENCES}

[1] S. Kwiatkowski, W. Barry, J. Julian, R. Low, D. Plate, G. Portmann, D. Robin , "Camshaft Bunch Kicker Design for the ALS Storage Ring” EPAC'06. 